\title{
Images - Penile pain in the setting of end-stage renal disease: An unusual anatomic location for calciphylaxis
}

\author{
Ummah Salma Nisar, MBBS ${ }^{1}$; John C. Cheville, $\mathrm{MD}^{2}$; Charles D. Sturgis, MD ${ }^{2}$ \\ ${ }^{1}$ Liver Transplant Center, Mayo Clinic, Rochester, MN, United States; ${ }^{2}$ Anatomic Pathology, Mayo Clinic, \\ Rochester, MN, United States
}

Cite as: Nisar US, Cheville JC, Sturgis CD. Images - Penile pain in the setting of end-stage renal disease: An unusual anatomic location for calciphylaxis. Can Urol Assoc J 2021 December 21; Epub ahead of print. http://dx.doi.org/10.5489/cuaj.7618

Published online December 21, 2021

Corresponding author: Dr. Charles D. Sturgis, Mayo Clinic Pathology, Rochester, MN, United States; Sturgis.Charles@Mayo.edu

$* * *$

\begin{abstract}
In this report, we present a case of penile calciphylaxis, an extremely rare and serious condition occurring in association with dysregulation of systemic calcium metabolism in the setting of chronic renal impairment. Calciphylaxis can occur at various body sites and is associated with diffuse vascular calcifications in small and medium-sized arteries of the involved tissues. Penile calciphylaxis has a grim prognosis. Calciphylaxis is an important etiologic differential diagnosis for penile necrosis and penile pain in patient's being treated with dialysis for end-stage kidney disease. Diagnosis of penile calciphylaxis is possible via clinical and radiological evaluations. Medical management may alleviate symptoms; however surgical interventions may be necessary, and histological studies may allow for definitive classification.
\end{abstract}

\section{Introduction}

Calciphylaxis (also called calcific uremic arteriolopathy) is a rare but serious condition associated with significant calcification of the media of small and medium sized arteries, primarily affecting the distal extremities ${ }^{1}$. Calciphylaxis is almost always seen in patients with end-stage renal disease who are on dialysis ${ }^{1-3}$. It is sometimes associated with hyperparathyroidism and elevated serum calcium and phosphorus levels ${ }^{4}$. Penile calciphylaxis is very rare and is associated with a poor prognosis. As the penis contains a rich network of interconnecting vascular channels, diseases of the vessels (such as calciphylaxis), may be associated with serious clinical symptoms. Patients with penile calciphylaxis often present with severe penile pain and necrosis of surrounding skin and subcutaneous tissues ${ }^{5}$. In this report, we 
present findings from an adult patient with penile calciphylaxis who underwent penectomy and survived for 5 weeks after the diagnosis of calciphylaxis was confirmed. Early diagnosis may increase survival time ${ }^{6}$.

\section{Case report}

A 60-year-old white man presented to the emergency department with urinary retention and penile pain. His symptoms began 20 days prior, when he presented to outpatient with pain in his digits and penis, with associated skin necrosis. He had a history of coronary artery disease, peripheral vascular disease, and end stage renal disease maintained on peritoneal dialysis after failed renal transplantation. Examination confirmed scrotal erythema and edema over necrotic tissue in the glans penis. Labs revealed serum calcium $8.1 \mathrm{mg} / \mathrm{dL}$, elevated serum phosphorus 6.9 $\mathrm{mg} / \mathrm{dL}$, elevated PTH $114 \mathrm{pg} / \mathrm{mL}$, and elevated WBC 17.2 x 10(9)/L. Imaging was suspicious for superinfection of the penis with gas identified in the soft tissues. A suprapubic catheter was placed after consultation with a urologist, followed by partial penectomy, with penile and scrotal debridement. Calciphylaxis was clinically / radiologically somewhat favored over Fournier's gangrene due to lack of subcutaneous emphysema and hemodynamically stable state. Gross examination of the penectomy showed gray-blue discoloration, softening and sloughing of surface layers of tissue with prominent vascular calcifications (Figure 1). Histologically, extensive necrosis of the central shaft was identified in a roughly triangular shape with large expanses of corpus cavernosum being extensively degenerated with intravascular and sheet like calcifications spanning the entirety of tissue, sparing the urethra, corpus spongiosum and inferior vascular structures (Figure 2). Superficial secondary bacterial overgrowth was noted. Postoperatively, the patient was managed with supportive care. He died 5 weeks later at his home under hospice care.

\section{Discussion}

Calciphylaxis is a rare condition involving calcification of small and medium arteries and is most commonly encountered in the distal extremities ${ }^{1-5}$. Calciphylaxis is seen in $1-4 \%^{2,3}$ of patients with end stage renal disease and has a grim prognosis. Penile involvement is uncommon, with only rare reported cases and small series in the English language literature ${ }^{5-11}$. Most patients who present with penile calciphylaxis may also have comorbidities like atherosclerosis, peripheral vascular disease, diabetes, hypertension, and obesity ${ }^{5}$. Control of these comorbid conditions in patient with end stage renal disease may diminish the likelihood of developing calciphylaxis. Patients with penile calciphylaxis typically present with penile pain and necrosis of surrounding skin and subcutaneous tissue. The mean survival after diagnosis is 2.5 months, with maximum survival generally not exceeding 6 months ${ }^{9}$. Some authors suggest that early diagnosis and interventions may increase survival ${ }^{8}$. Aberrant serum calcium, phosphorus and parathyroid hormone levels seen in patients with penile pain in the setting of end stage renal disease may suggest a diagnosis of penile calciphylaxis, but serum parameters may in some instances fall within normal ranges. Radiological studies using modalities like Doppler, CT or MRI may 
contribute to diagnosis ${ }^{10}$. Doppler testing to evaluate the flow in penile blood vessels may be of supplemental value to MRI which can be helpful in delimiting boundaries between viable and necrotic tissue ${ }^{11}$. Treatments can be medical, surgical or both. Medical management may include administration of calcium and phosphate chelators to normalize elevated levels. For cases with associated hyperparathyroidism, cinacalcet administration and/or parathyroidectomy can be considered. Many patients ultimately undergo partial or complete penectomy to avoid further medical decompensation, significant pain and sepsis ${ }^{5}$. No significant differences in outcomes (mortality rates) are reported between medically and surgically managed patients.

\section{Conclusions}

Penile calciphylaxis should be considered in patients with end stage renal disease who present with ulcers or pain in the penis, particularly the glans. Patients who are being dialyzed and/or who have concomitant diabetes are at greatest risk. The diagnosis can generally be reached using clinical, biochemical and radiological evaluations. Tissue confirmation may be achieved with biopsy (which has attendant risks) or by examination of penectomy specimens. Penile calciphylaxis in the setting of end stage renal disease is associated with unfavorable prognosis. Survival is most often measured in weeks to months. 


\section{References}

1. Kent, R. B., 3rd, \& Lyerly, R. T. (1994). Systemic calciphylaxis. Southern medical journal, 87(2), 278-28

2. Ivker RA, Woosley J, Briggaman RA. Calciphylaxis in three patients with end-stage renal disease. Arch Dermatol 1995;131(1):63-68

3. Vedvyas C, Winterfield LS, Vleugels RA. Calciphylaxis: a systematic review of existing and emerging therapies. J Am Acad Dermatol. 2012;67:253-260

4. Duh, Q. Y., Lim, R. C., \& Clark, O. H. (1991). Calciphylaxis in secondary hyperparathyroidism. Diagnosis and parathyroidectomy. Archives of surgery (Chicago, Ill. : 1960), 126(10), 1213-1219

5. Wood JC, Monga M, Hellstrom WJ. Penile calciphylaxis. Urology 1997;50(4):622-624

6. El-Taji, O., Bondad, J., Faruqui, S., \& Bycroft, J. (2020). Penile calciphylaxis: a conservative approach. Annals of the Royal College of Surgeons of England, 102(2), e36e3

7. Tezuka M, Mizusawa H, Tsukada M, et al. Severe necrosis of the glans penis associated with calciphylaxis treated by partial penectomy. IJU Case Rep 2020;3(4):133-136

8. Gomes Torres JH, Neves Ribeiro SC, Carvalho de Souza I, Fernandes de Almeida Hellebrandt MC, Budib LJ, Freitas Filho LG. Penile necrosis and calciphylaxis. Urol Case Rep. 2021;39:101770

9. Karpman, E., Das, S., \& Kurzrock, E. A. (2003). Penile calciphylaxis: analysis of risk factors and mortality. The Journal of urology, 169(6), 2206-2209.

10. Morrison, M., Merati, M., Ramirez, J., Cha, H. C., \& LaFond, A. (2016). Penile calciphylaxis diagnosed with computed tomography. Journal of the European Academy of Dermatology and Venereology : JEADV, 30(2), 352-353

11. Campbell, R. A., Alzweri, L. M., Sopko, N. A., Macura, K. J., \& Burnett, A. L. (2017). Penile Calciphylaxis: The Use of Radiological Investigations in the Management of a Rare and Challenging Condition. Urology case reports, 13, 113-116 


\section{Figures and Tables}

Fig. 1. Serially sectioned penectomy specimen with peripherally sloughed skin showing calcification of cavernous arteries (best seen in the left most section) with necrosis of corpora cavernosa and peripheral sparing. Necrosis extends to dorsal aspect of urethra involving corpora spongiosa.

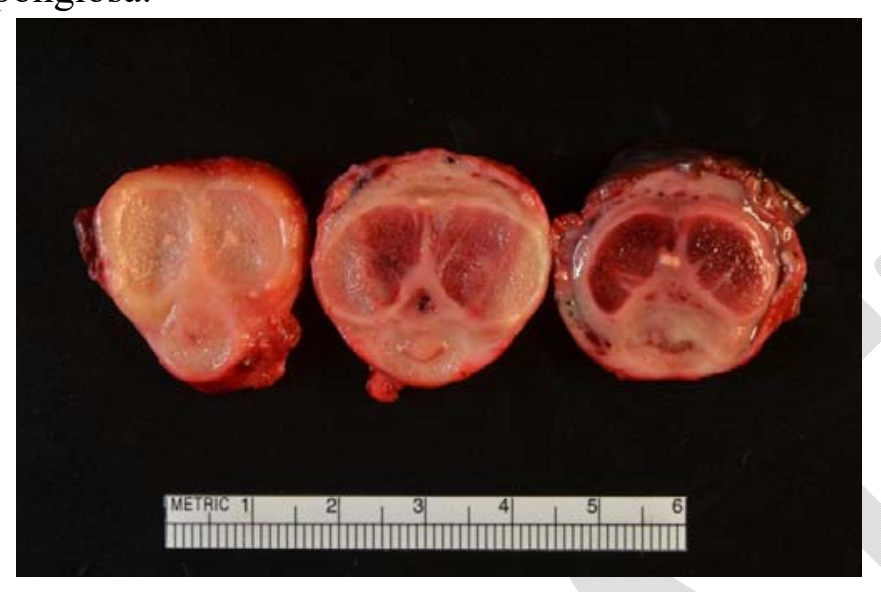

Fig. 2. Histology of the penectomy specimen showing complete necrosis of corpora cavernosa (2A, Hematoxylin and Eosin, 100X) with diffuse granular calcification throughout soft tissues (2B, Von Kossa, 400X). Urethral artery with coarse intimal calcification and necrosis with inflammation intervening between vasculature and corpus spongiosum $(2 \mathrm{C}$, Hematoxylin and Eosin, 40X) dorsolateral corpus spongiosum with necrosis showing relative sparing of urethra proper and inferior soft tissues (2D, Hematoxylin and Eosin, 40X).

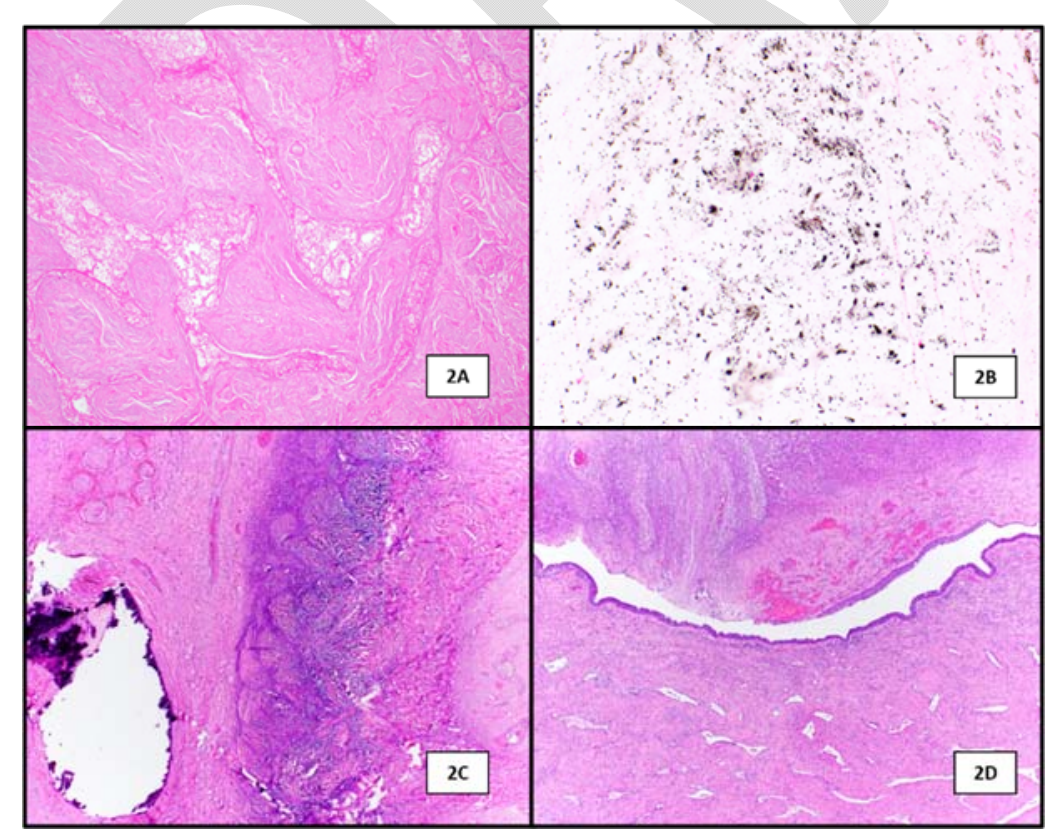

